\title{
Implementation and Analysis of Threshold Sensitive Stable Election Protocol
}

\author{
Shailie Rai ${ }^{1}$, Pallavi Gupta ${ }^{2}$ \\ M. Tech Scholar, Department of Electronics and Communication, Babu Banarasi Das University, Lucknow, India ${ }^{1}$ \\ Assistant Professor, Department of Electronics and Communication, Babu Banarasi Das University, Lucknow, India ${ }^{2}$
}

\begin{abstract}
The multitude of possible applications supported by wireless sensor networks (WSNs) has generated much interest from the research community. The recent advancement in technology have enabled the development of low cost, energy-efficient and versatile wireless sensing devices. The wireless sensor networks (WSNs) have dynamic and distributed nature which introduces very special requirements for the routing protocols that should be fulfilled. The most significant feature of a routing protocol is the energy efficiency and the extension of the network lifetime, in order to be efficient for WSNs. The Threshold Sensitive Stable Election Protocol (TSEP) is a three level heterogeneous reactive routing protocol. The energy dissipation is reduced due to energy heterogeneity and prolongs network lifetime, increases the stability period and throughput.
\end{abstract}

Keywords: Wireless sensor networks, routing protocols, threshold sensitive stable election protocol, graphical user interface, heterogeneous environment.

\section{INTRODUCTION}

Wireless Sensor Networks (WSNs) is a network of small battery powered sensor nodes whose task is to sense a phenomenon in a cooperative manner as well as controlling the environment [1]. These sensor nodes are densely deployed in a sensor field (monitoring area), they monitor the collected data and forwards to other sensor nodes by the hopping mechanism. These sensor nodes sense data from remote areas and transfer their report to the base station (BS) also known as sink. In WSNs deployment of nodes in an area can be through random or proper deployment mechanism, these nodes can be stationary or mobile. WSNs are an amalgamation of various technologies such as sensing, embedded computation and information processing; it finds its wide usage in numerous fields like habitat and environment monitoring, battlefield surveillance, medical diagnoses, traffic management and many other fields [2, 3].

The major objective of these networks is to have accuracy, flexibility, reliability, ease in deployment and economical. The network lifetime is limited as sensor nodes are battery operated and it is difficult to recharge batteries at a regular basis because the sensor nodes are deployed in inaccessible areas. Therefore, energy-efficiency and enhancement of network lifetime has been a main issue in the research field.

In order to achieve enhanced network lifetime and reduced power consumption many energy-efficient routing protocols have been developed, in varying application areas. Placing few heterogeneous nodes in wireless sensor network is an effective way to increase network lifetime and reliability [4]. The three general categories of resource heterogeneity in a sensor node are energy heterogeneity, computational heterogeneity and link heterogeneity [5]. Heterogeneity in terms of energy signifies that the heterogeneous sensor node is line powered or it has replaceable battery.

When the heterogeneous node has a more powerful microprocessor and additional memory than the normal node it is considered as a case of Computational heterogeneity. Thus the heterogeneous nodes can offer longer-term storage and complex data processing. When the heterogeneous node has long-distance network transceiver and high-bandwidth than the normal node it is considered as a case of Link heterogeneity, providing more reliable data transmission. Among above three types of resource heterogeneity, energy heterogeneity is the most important heterogeneity as in both computational and link heterogeneity there is more consumption of energy resource.

In order to prolong the network lifetime clustering of sensor nodes is an efficient solution. As it provides various benefits such as scalability, load balancing, and reduction in collisions during intra-cluster communication [6]. Cluster head selection schemes can be divided into two categories: Homogeneous and Heterogeneous schemes. In the homogeneous scheme, each sensor node in the network is equipped with the identical amount of energy such as LEACH [7], HEED [8]. The homogeneous scheme is not efficient for heterogeneous networks as the sensor nodes with low energy level can have a high probability of election than the nodes with high energy level. In the heterogeneous scheme, each sensor node has different amount of energy because each node is allotted with various task [4], [9] such as SEP [10], DEEC [11]. In this paper, we implement and analyze the TSEP protocol for heterogeneous wireless sensor networks (HWSNs). Javaid et al. proposed Threshold Sensitive Stable Election Protocol (TSEP) [12]. In the TSEP protocol, the cluster heads are selected on the basis of the threshold. This protocol considers three types of nodes that have different energy levels, 
called as the advance, intermediate and normal nodes. The initial energy for normal nodes is $\mathrm{E}_{0}$, for advanced nodes, $\mathrm{E}_{\mathrm{adv}}=\mathrm{E}_{0}(1+\alpha)$ and for intermediate nodes is $\mathrm{E}_{\mathrm{int}}=\mathrm{E}_{0}(1+\mu)$, where $\alpha$ and $\mu$ both are energy factor for advanced and intermediate nodes respectively and $\mu=\alpha / 2$. The probabilities to become normal, intermediate and advanced nodes are $\mathrm{P}_{\mathrm{nrm}}, \mathrm{P}_{\mathrm{int}}$ and $\mathrm{P}_{\mathrm{adv}}$ respectively as shown in (1), (2) and (3).

$$
\begin{gathered}
P_{n r m}=\frac{P_{o p t}}{1+m \cdot \alpha+b \cdot \mu} \\
P_{\mathrm{int}}=\frac{P_{o p t}(1+\mu)}{1+m \cdot \alpha+b \cdot \mu} \\
P_{a d v}=\frac{P_{o p t}(1+\alpha)}{1+m \cdot \alpha+b \cdot \mu}
\end{gathered}
$$

To ensure that cluster head selection is done as assumed above, the threshold level is taken into consideration. The threshold for normal, intermediate and advanced nodes are shown by (4), (5) and (6) respectively.

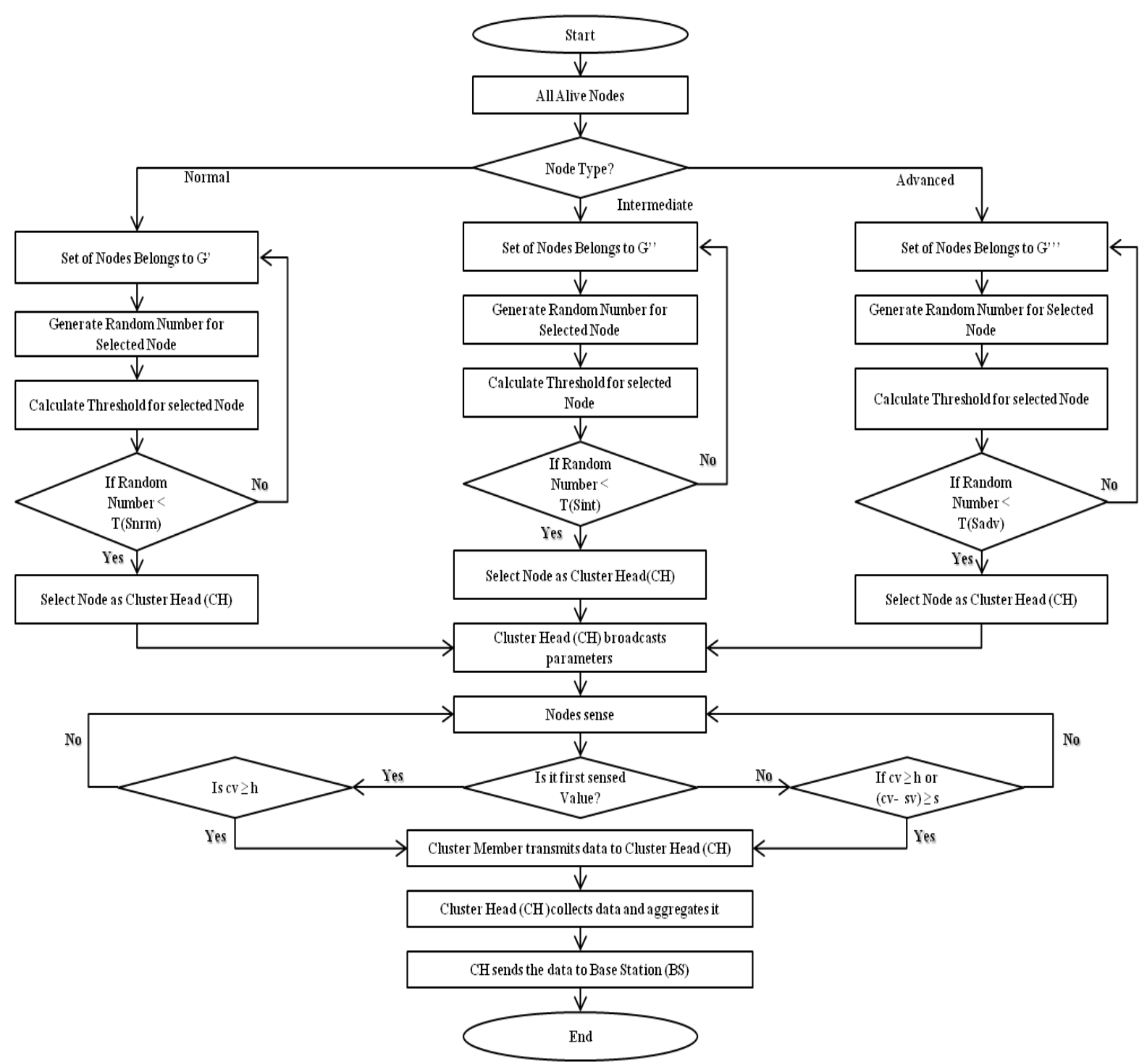

Fig. 1. Flowchart of TSEP Protocol 


$$
\begin{gathered}
T_{n r m}= \begin{cases}\frac{p_{n r m}}{1-p_{n r m}\left(r \cdot \bmod \frac{1}{p_{n r m}}\right)} & \text { if } \mathrm{n}_{n r m} \in G^{\prime} \\
0 & \text { otherwise }\end{cases} \\
T_{\text {int }}= \begin{cases}\frac{p_{\text {int }}}{1-p_{\text {int }}\left(r \cdot \bmod \frac{1}{p_{\text {int }}}\right)} & \text { if } \mathrm{n}_{\text {int }} \in G^{\prime \prime} \\
0 & \text { otherwise }\end{cases} \\
T_{a d v}= \begin{cases}\frac{p_{a d v}}{1-p_{a d v}\left(r \cdot \bmod \frac{1}{p_{a d v}}\right)} & \text { if } \mathrm{n}_{a d v} \in G^{\prime \prime} \\
0 & \text { otherwise }\end{cases}
\end{gathered}
$$

Where G', G" and G" "are the set of normal, intermediate and advanced nodes that have not become CHs in the last $1 / \mathrm{P}_{\text {nrm, }}, 1 / \mathrm{P}_{\text {int }}$ and $1 / \mathrm{P}_{\text {adv }}$ round respectively. The TSEP protocol increases the stability, lifetime of the network because of three levels of heterogeneity and it also increases the throughput of the network. As TSEP is a reactive routing protocol, thus the nodes keep on sensing continuously, but the transmission is not done in a frequent manner. Fig. 1. depicts the working of the TSEP protocol.

The remainder of this paper is organized as follows: Section 2 provides description of simulation setup. Section 3 provides results and analysis of TSEP protocol. Finally, Section 4 concludes the paper.

\section{SIMULATION SETUP}

The simulation setup of TSEP protocol is implemented in MATLAB. The Graphical User Interface (GUI) toolbox of the MATLAB provides a lucid representation of analysis of protocol. In the simulation the network size in $100 \times 100$ meters $^{2}$. The total of number of sensor nodes (n) is 100 . There are three types of nodes namely, normal, intermediate and advanced nodes which are randomly deployed. Thus, the horizontal and vertical coordinates of each sensor node are randomly selected between 0 and the maximum value of the dimension.

The assumptions for this setup are as follows: All the deployed sensor nodes are stationary, the wireless sensor network consists nodes with three different energy levels i.e. heterogeneous sensor nodes, the Base Station (BS) is located at the center of the network area and the distance measurement is based on the wireless radio signal power. The advance nodes have energy greater than all other nodes, intermediate nodes have energy in between normal and advance nodes while the remaining nodes are the normal nodes. The intermediate nodes are selected by using $b$, a proportion of nodes which are intermediate nodes and they have $\mu$ times more energy than that of normal nodes. The simulation parameters required for the implementation of TSEP protocol are shown in Table 1.

Table 1. Simulation Parameters

\begin{tabular}{|c|c|c|}
\hline Description & Parameters & Value \\
\hline Network Size & $\mathrm{M} \times \mathrm{M}$ & $100 \times 100$ \\
\hline Base Station Location & $\mathrm{BS}$ & $(50,50)$ \\
\hline Initial Energy of Nodes & $\mathrm{E}_{0}$ & $0.5 \mathrm{~J}$ \\
\hline Energy dissipated per bit & $\mathrm{E}_{\mathrm{elec}}$ & $50 \mathrm{~nJ} / \mathrm{bit}$ \\
\hline Transmit amplifier if $\mathrm{d}_{\mathrm{toBS}} \leq \mathrm{d}_{0}$ & $\varepsilon_{\mathrm{fs}}$ & $10 \mathrm{pJ} / \mathrm{bit} / \mathrm{m}^{2}$ \\
\hline Transmit amplifier if $\mathrm{d}_{\mathrm{toBS}}>\mathrm{d}_{0}$ & $\varepsilon_{\mathrm{mp}}$ & $0.0013 \mathrm{pJ} / \mathrm{bit} / \mathrm{m}^{4}$ \\
\hline Data aggregation energy by $\mathrm{CH}$ & $\mathrm{E}_{\mathrm{DA}}$ & $5 \mathrm{~nJ} / \mathrm{bit} / \mathrm{message}$ \\
\hline Number of Nodes & $\mathrm{n}$ & 100 \\
\hline Proportion of advanced nodes & $\mathrm{m}$ & 0.1 \\
\hline Energy factor for Advanced nodes & $\alpha$ & 1 \\
\hline Optimal Probabaility & $\mathrm{P}_{\mathrm{opt}}$ & 0.1 \\
\hline Size of data packet & $\mathrm{K}$ & $4000 \mathrm{bits}$ \\
\hline
\end{tabular}


The Fig. 2. shows the Graphical User Interface (GUI) for the TSEP protocol. This GUI has a panel with the title "Enter the Values in the field below:"'and has four text and edit text boxes, also two push buttons. The names of four text boxes are as follows: X-Axis, Y-Axis, No. of Nodes (n) and No. of rounds (rmax). In the four edit text boxes of the GUI the values can be entered. The two push buttons are Execute and Clear Values. With the Execute button the execution starts and the Clear Values button clears all the values in the edit text boxes and also clears all axes. There is a text box named as "TSEP : First Dead Node at Round" and a edit text box which displays N/A until the round number at which the first node becomes dead is obtained on the extreme left lower corner of the GUI. There are five axes to display the results. When the execution starts firstly a random sensor network is created for the TSEP protocol which consists of three types of nodes and base station (sink) on the first axes. The representations of different types of nodes and base station (sink) for the Graphical User Interface (GUI) are as follows:

\section{Normal Node: o \\ Intermediate Node: \\ Advanced Node: + \\ Base Station: $x$}

The election of Cluster Heads (CHs) for the Threshold Sensitive Stable Election Protocol (TSEP) starts in the random sensor network on the second axes. The cluster head selection is done on the basis of the probability of a node to become cluster head. The optimal probability of nodes, which are divided on the basis of energy, to be elected as a $\mathrm{CH}$ can be calculated by using following formulas given in (1), (2) and (3). After the completion of maximum number of rounds, the plots for the number of dead nodes per round, number of alive nodes per round and the packets sent to the base station are displayed on the third, fourth and fifth axes respectively. The push button named as Close, is used to exit from the GUI. In this simulation it is assumed that the values for the four edit text boxes under the panel are as follows: X-Axis $=100$, Y-Axis $=100$, No. of Nodes $(n)=100$ and No. of rounds $(\operatorname{rmax})=12000$.

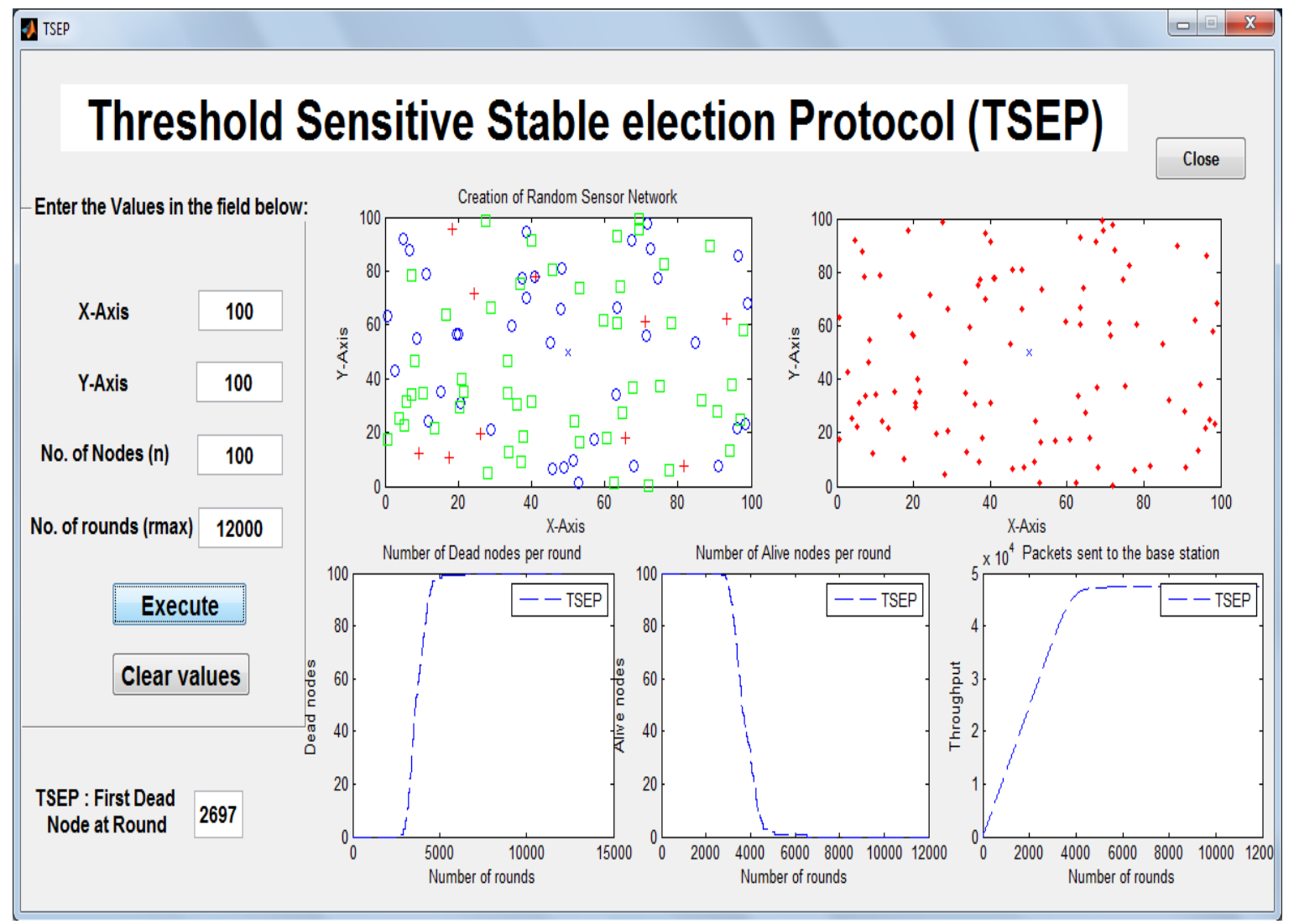

Fig. 2. Graphical User Interface (GUI) for the TSEP Protocol

\section{III.RESULT AND ANALYSIS}

Using the simulation setup, the performance of TSEP is observed. To simulate the Threshold Sensitive Stable Election Protocol in MATLAB, the functions are created in the editor window. Under the function, the parameters or variables are 
declared. The parameters to be initialized here are $x$-axis coordinates, $y$-axis coordinates, number of nodes and number of rounds.Various functions are created to perform numerous tasks. This function declaration is called the MATLAB Code. An easy representation of the protocol is obtained through MATLAB GUI. The text buttons and push buttons works under Call back functions which is coded in M-file for GUI. There are four text buttons for entering the values for $\mathrm{x}$-axis coordinates, $\mathrm{y}$-axis coordinates, number of nodes (n) and number of rounds (rmax). Other three buttons are the push buttons to start the execution, clear all the values in the fields and exit from the GUI. It is clear from the Fig. 3. that the number of dead nodes starts increasing after 2697th round and up to 3609th round 50\% of the nodes are dead. All nodes are dead at round $6522 \mathrm{nd}$.

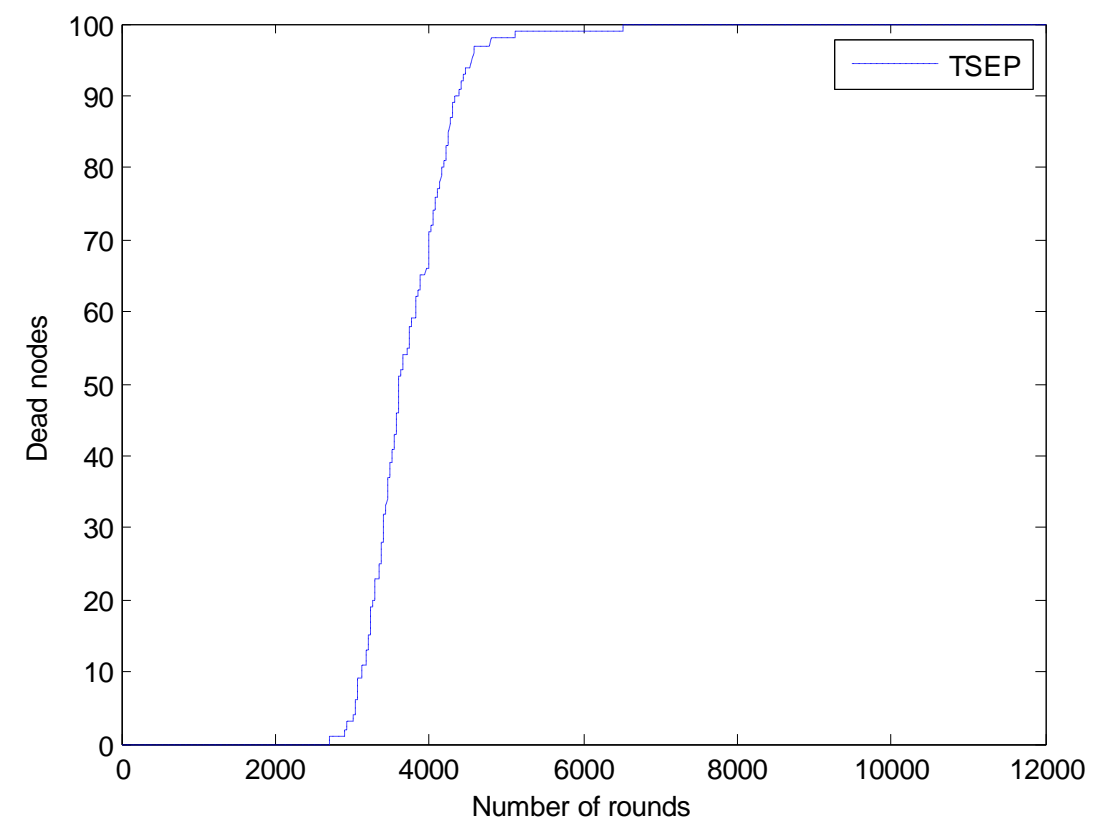

Fig. 3. Number of Dead nodes per round

Based on the network features and parameters, the protocol was implemented and examined using MATLAB GUI. Fig. 4. shows the number of alive nodes for TSEP protocol. Table 2 shows that in TSEP the first node died at 2697th round. Hence the stability region of the network is significantly increased. Similarly, 50\% nodes died at the 3609th round. The network is working for a longer period of time because the last node died at the 6522nd round in TSEP.

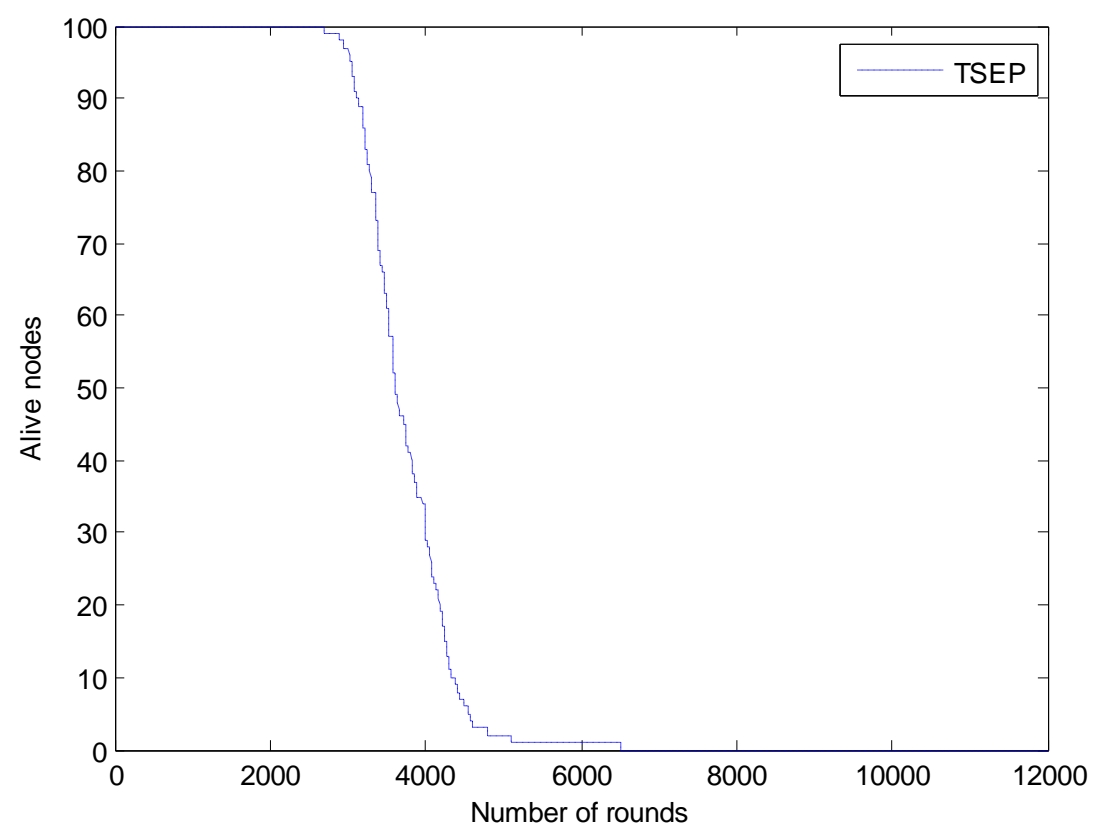

Fig. 4. Number of Alive nodes per round 
Table 2 . Comparison of dead nodes

\begin{tabular}{|c|c|}
\hline \multirow{2}{*}{ \% Nodes } & Number of rounds \\
\cline { 2 - 2 } & TSEP \\
\hline $1 \%$ & 2697 \\
\hline $50 \%$ & 3609 \\
\hline $100 \%$ & 6522 \\
\hline
\end{tabular}

Fig. 5. represents the packets sent to the base station or sink in each round of the TSEP protocol.

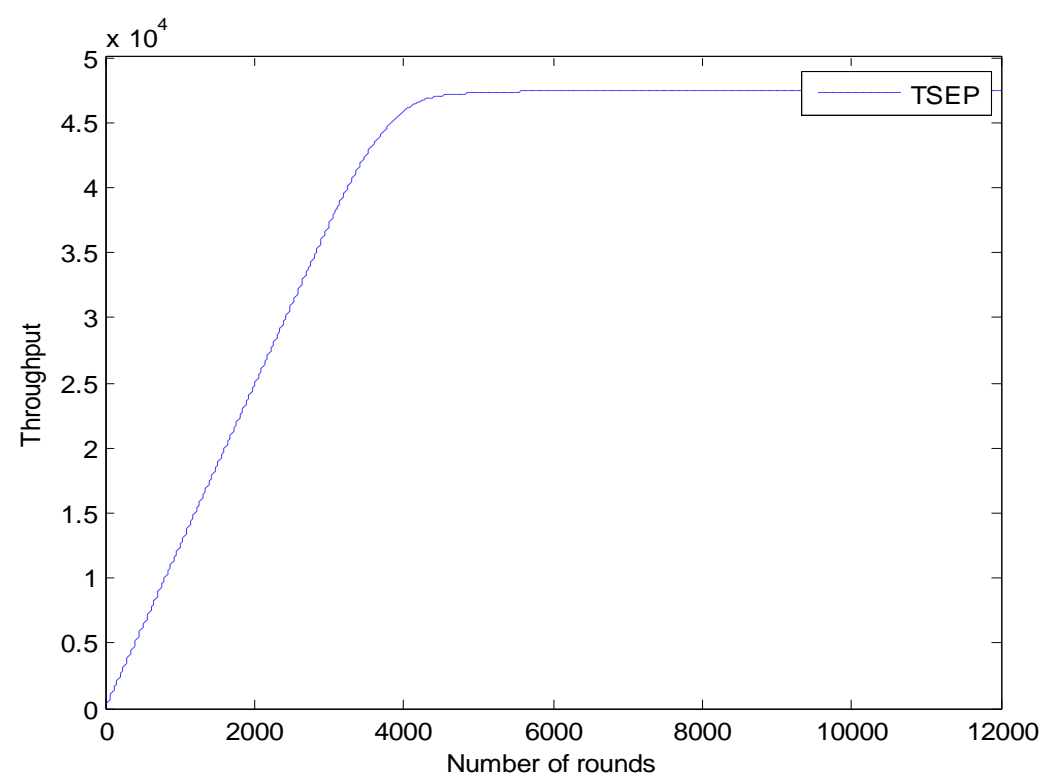

Fig. 5. Throughput of the protocol

Table 3 shows the performance analysis table of TSEP which clearly specifies that it is an efficient routing protocol with enhanced stability and instability period, improved lifetime and throughput.

Table 3. Performance Analysis table of TSEP

\begin{tabular}{|c|c|c|c|c|}
\hline Protocol & Stability Period & Instability period & Lifetime & Throughput \\
\hline TSEP & 2697 & 3825 & 6522 & 47,510 \\
\hline
\end{tabular}

Fig. 6. Describes the Half Node Dead (HND) versus Last Node Dead (LND) performance of TSEP protocol

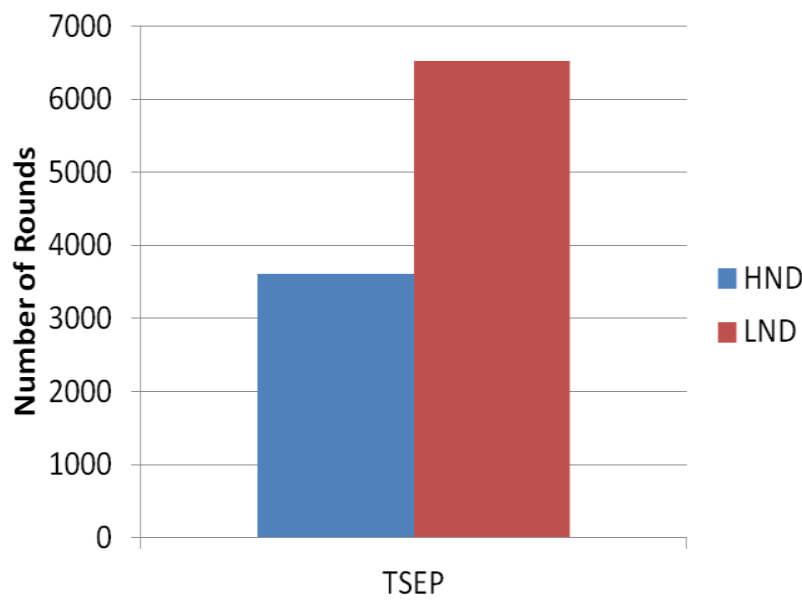

Fig. 6. HND versus LND of TSEP protocol 
Fig. 7. describes the stability versus instability period of the TSEP protocol.

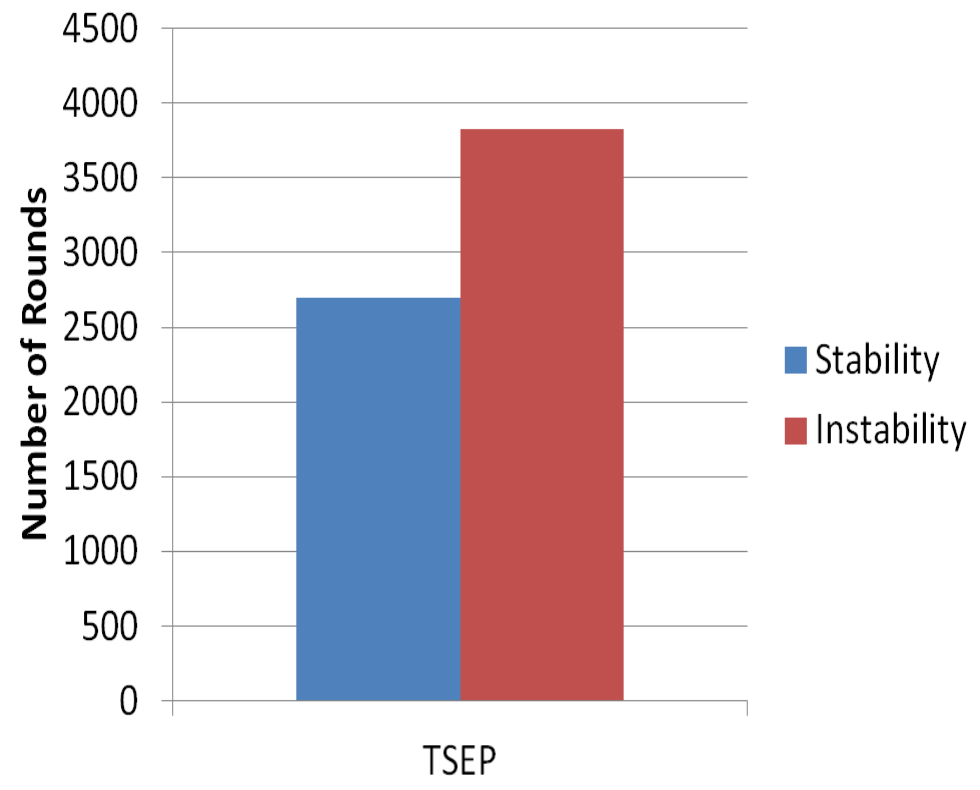

Fig. 7. Stability versus Instability period of TSEP protocol

\section{IV.CONCLUSION}

In this paper, a Graphical User Interface (GUI) for the TSEP protocol is presented. The recapitulations of this study are as follows: TSEP is a reactive routing protocol and it has nodes with three different levels of energies. The cluster heads (CHs) selection is threshold based because of three levels of heterogeneity. The performance of TSEP protocol is analysed on the basis of half node dead (HND) versus last node dead (LND) and stability versus instability period. The simulation results have demonstrated the efficiency and reliability and of this approach. It was observed that TSEP is a robust protocol as it increases the stability period and provides enhanced network lifetime and throughput.

\section{REFERENCES}

[1] I. Akyildiz, W. Su, Y. Sankarasubramaniam, and E. Cayirci, "A survey on sensor networks," IEEE Communications Magazine, vol. 40, no. 8, pp. 102-114, August 2002

[2] I.F. Akyildiz, W.J. Su, Y. Sankarasubramaniam, E. Cayirci, "Wireless sensor networks: a survey", Computer Networks 38, 393-422, 2002.

[3] J.N. Al-Karaki, A.E. Kamal, "Routing techniques in wireless sensor networks: a survey", IEEE Wireless Communications, 2004.

[4] D. Kumar, T. C. Aseri, and R. B. Patel, "EEHC: Energy efficient heterogeneous clustered scheme for wireless sensor networks", Computer Communications, 32(4), 662-667, 2009.

[5] M. Yarvis, N. Kushalnagar, H. Singh, "Exploiting heterogeneity in sensor networks", IEEE INFOCOM, 2005.

[6] S. Gajjar, M. Sarkar and K. S. Dasgupta, "Cluster Head Selection Protocol using Fuzzy Logic for Wireless Sensor Networks", International Journal of Computer Applications, 38-43,2014.

[7] W. R. Heinzelman, A. Chandrakasan, and H. Balakrishnan, "Energy-efficient communication protocol for wireless microsensor networks", System Sciences. In Proceedings of the 33rd annual Hawaii international conference, pp. 4-7, 2000.

[8] O. Younis, S. Fahmy, "Heed: a hybrid, energy- efficient distributed clustering approach for Ad hoc sensor networks," IEEE Trans on mobile computing, 3(4), pp.660-669, 2004.

[9] P. Saini, A. K. Sharma, "Energy efficient scheme for clustering protocol prolonging the lifetime of heterogeneous wireless sensor networks", International Journal of Computer Applications, 6(2), 30-36, 2010.

[10] G. Smaragdakis, I. Matta, and A. Bestavros, "SEP: A stable election protocol for clustered heterogeneous wireless sensor networks," Second International Workshop on Sensor and Actor Network Protocols and Applications, July 2004

[11] L.Qing, Q. X.Zhu, and M. W.Wang, "A distributed Energy-Efficient Clustering Algorithm for Heterogeneous Wireless Sensor Networks," Journal of Software, Vol.17, No. 3, pp. 481-489, 2006.

[12] A. Kashaf, N. Javaid, Z.A. Khan and I.A. Khan, "TSEP: Threshold-Sensitive Stable Election Protocol for WSNs", IEEE 10th International Conference on Frontiers of Information Technology, pp. $164-168,2012$. 